\title{
DOE, formulation, and optimization of Repaglinide nanostructured lipid carriers
}

\author{
Shady A. Swidan ${ }^{1 *}$, Zeinab N. Mansour ${ }^{2}$, Zeinab A. Mourad², Nahla A. Elhesaisy², Nada A. Mohamed², Mohamed S. Bekheet ${ }^{2}$, \\ Mohamed A. Badawy ${ }^{2}$, Mai M. Elsemeiri², Aya E. Elrefaey², Amera M. Hassaneen² \\ ${ }^{1}$ Department of Pharmaceutics, Faculty of Pharmacy, The British University in Egypt, El-Sherouk City, Egypt. \\ ${ }^{2}$ Undergraduate student of the 5th year, Faculty of Pharmacy, The British University in Egypt, El-Sherouk City, Egypt.
}

\begin{tabular}{l}
\hline ARTICLE INFO \\
\hline Received on: 09/07/2018 \\
Accepted on: 23/08/2018 \\
Available online: $31 / 10 / 2018$ \\
\\
\hline Key words: \\
Nanostructured lipid \\
carriers, emulsification-- \\
ultrasonification, Repaglinide, \\
optimization, DSC.
\end{tabular}

\begin{abstract}
Nanostructured lipid carriers (NLC) are a recent approach for the delivery of poorly soluble drugs with low oral bioavailability. The oral antidiabetic Repaglinide (RPG) was loaded into NLC using emulsification-ultrasonification technique. A design of experiment was constructed to study the formulation variables. The influence of the liquid lipid to the solid lipid ratio and the concentration of the surfactant on mean particle size, zeta potential, and drug entrapment efficiency was demonstrated. The mean particle size ranged from $182 \pm 7.9 \mathrm{~nm}$ to $452 \pm 66.1 \mathrm{~nm}$. All particles were negatively charged and the zeta potential values ranged from $-7.9 \pm 0.9 \mathrm{mV}$ to $-44.4 \pm 6.2 \mathrm{mV}$. The highest entrapment efficiency was obtained with the minimum solid lipid to liquid lipid ratio and lowest surfactant concentration. All RPG-NLC formulae showed biphasic time-dependent in vitro release and the studied factors were optimized and the optimum formula was evaluated for in vitro release and crystallinity. The in vitro release of the optimized formula fitted to the Higuchi diffusion model. In conclusion, this study showed the potential of NLC as a carrier for controlled release of RPG.
\end{abstract}

\section{INTRODUCTION}

Diabetes mellitus (DM) is an important chronic disease, which may cause severe complications if it is not properly controlled (He et al., 2015). Those severe complications include cardiovascular problems, high blood pressure, impaired lipid profile, nephropathy, neuropathy, and retinopathy. This makes this disease the seventh worldwide leading cause of death (Kramer et al., 2013). DM has two types and type II DM is the most common type and accounts for more than $90 \%$ of the cases (Zhu et al., 2013).

Different classes of oral hypoglycemic agents are used for the treatment of type II DM. Repaglinide (RPG), $(S)(+)-2$ ethoxy4-(2[[3-methyl-1[2-(1-piperidinyl)phenyl]-butyl]amino]-2

"Corresponding Author

Shady A. Swidan, Department of Pharmaceutics, Faculty of Pharmacy,

The British University in Egypt, El-Sherouk City, Egypt.

E-mail: shady.swidan@bue.edu.eg oxoethyl) benzoic acid, is a new potent blood glucose lowering agent (Figure 1) (Akhtar et al., 2013). It belongs to the class of carbamoyl-methyl benzoic acids. It acts by stimulation of the release of insulin from the pancreatic cells by blocking of ATPsensitive potassium channels in the plasma membrane (Hu et al., 2000). Although RPG has an excellent antidiabetic effect, it suffers from certain problems, which diminish its activity and require frequent administration. RPG has a short half-life (about 1 hour), it suffers from a significant first-pass effect and it has a low oral bioavailability of about $50 \%$ (Kassem et al., 2017).

In order to increase the patient compliance and enhance its oral bioavailability, different RPG nanoparticles were formulated such as nanoemulsions (Akhtar et al., 2016), self-nano emulsifying systems (Kamble et al., 2013), nanocrystals (Gadadare et al., 2015), and solid lipid nanoparticles (Ebrahimi et al., 2015). One of the most promising novel nano-based techniques is nanostructured lipid carriers (NLC). NLC is a new improved generation of solid lipid nanoparticles (SLN), in which a spatially incompatible liquid lipid is added to the solid lipid to form less ordered 


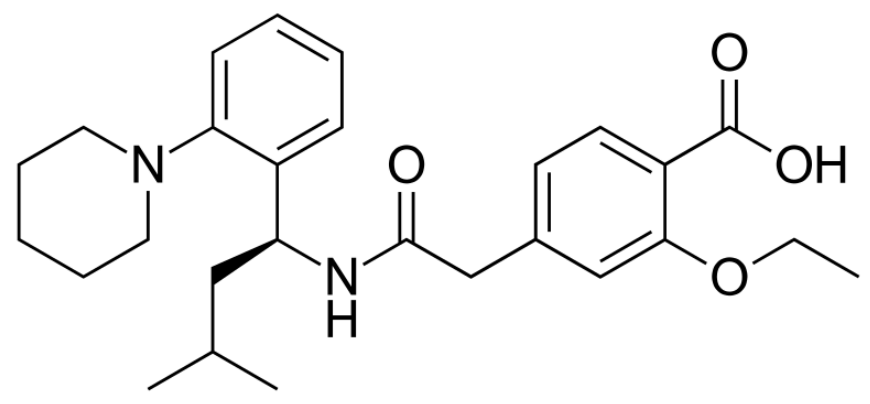

Figure 1. Chemical structure of RPG.

lipid nanoparticles (Swidan et al., 2016). It overcomes the disadvantages of SLN, including the low entrapment efficiency and drug loading, especially upon storage due to the drug expulsion from the highly ordered solid lipid crystals. The presence of the liquid lipid disrupts the symmetry of the lipid crystals and shows imperfection of the crystals, thus allowing more drug to be incorporated and lower amount to leave the crystals upon storage while maintaining the solid state at both room and body temperatures (Weber et al., 2014).

Several methods can be used for the preparation of NLC such as microemulsion, solvent diffusion, solvent injection, double emulsion, and emulsification ultrasonification (Das and Chaudhury, 2011). In this study, emulsification-ultrasonification method was used as it has a number of advantages; it avoids the use of organic solvents, which may cause toxicity, it is also simple and leads to the formation of small and uniform nanoparticles.

Different factors affect the shape, size, and the function of NLC such as types of solid lipids and liquid lipids, the ratio of both lipids to each other and to the drug, the type and concentration of surfactants, and other different factors related to the preparation technique (Tan et al., 2010; Yang et al., 2014).

In this study, the objective was to apply response surface methodology optimization model to optimize RPG loaded NLC. Two formulation factors were selected as independent variables. The prepared formulae were characterized by the determination of particle size (PS), polydispersibility index (PDI), and zeta potential (ZP). Entrapment efficiency (EE\%), drug loading (DL\%), and in vitro RPG release were evaluated and the optimized formula was evaluated with the same experiments and the crystallinity of the optimized form was studied using differential scanning calorimetry (DSC). The shape and morphology of the optimized formula were examined using a transmission electron microscope (TEM).

\section{MATERIALS AND METHODS}

\section{Materials}

RPG and the solid lipid glyceryl monostearate (GMS) are kind gifts from EIPICO pharmaceutical company. Oleic acid (OA) was purchased from Chemajet (Egypt). Polyoxyethylenesorbitan monooleate (Tween 80) - the used surfactant-was purchased from Acros (Netherlands). Potassium dihydrogen phosphate and sodium hydrogen phosphate were purchased from Lobechem (India). All other chemicals and solvents are of pharmaceutical grade and were used with no further modifications.

\section{Methods}

\section{Experimental design}

Two independent variables were selected for the study, the ratio of the liquid lipid to the solid lipid and the concentration of surfactant. Each factor has three levels: high, medium, and low. The dependent variables or the responses to be determined are the mean PS, ZP and EE\%. Values and constraints of both independent variables and responses are listed in Table 1. A $3^{2}$ factorial design was done and the composition of the nine resulted formulae is listed in Table 2. The optimization was done by response surface methodology using Statgraphics ${ }^{\circledR}$ centurion XVII software.

\section{Preparation of RPG loaded (NLCs)}

The RPG-NLCs formulae were prepared by emulsification and ultrasonification technique (Das et al., 2011) with few modifications. The organic phase-composed of the calculated weights of RPG, the liquid lipid OA, and the solid lipid GMS - was melted by heating up to $5^{\circ} \mathrm{C}$ above the melting point of GMS. The aqueous phase was prepared by dissolving tween 80 in water and heated to the same temperature as that of molten lipid phase. The preheated aqueous solution was added to the molten lipid phase and homogenized using WiseTis homogenizer HG-15D (Diahan sci, South Korea) for 5 minutes at 10,000 rpm. The coarse hot $\mathrm{O} / \mathrm{W}$ emulsion obtained was sonicated using a probe sonicator for 1 minute with a pause for 30 seconds for two cycles at $50 \mathrm{~W}$ (Vibra cell, Sonics, USA). The obtained NLCs were cooled to room temperature and stored at $4^{\circ} \mathrm{C}$.

Table 1. Values of independent and dependent variables.

\begin{tabular}{lccc}
\hline & \multicolumn{3}{c}{ Levels } \\
\cline { 2 - 4 } & Low (\%) & Medium (\%) & High (\%) \\
\hline Independent variables & & 30 & 45 \\
$X_{1}$ Liquid lipid ratio (to the solid lipid) & 15 & 1.5 & 2 \\
$X_{2}$ Surfactant concentration & 1 & Constraints \\
Dependent variables & \multicolumn{3}{|}{ Minimize } \\
$Y_{1}$ Particle size & \multicolumn{3}{|c}{ Maximize } \\
$Y_{2}$ Zeta potential & \multicolumn{3}{|c}{ Maximize } \\
$Y_{3}$ Entrapment efficiency & \multicolumn{3}{c}{} \\
\hline
\end{tabular}

Table 2. Composition of the prepared NLC formulae.

\begin{tabular}{cccccc}
\hline Form. & RPG (\%) & Lipids (\%) & S:L* & Tween 80 (\%) & Water (ml) \\
\hline F1 & 0.05 & 2 & $85: 15$ & 1.5 & 20 \\
F2 & 0.05 & 2 & $85: 15$ & 2 & 20 \\
F3 & 0.05 & 2 & $70: 30$ & 2 & 20 \\
F4 & 0.05 & 2 & $85: 15$ & 1 & 20 \\
F5 & 0.05 & 2 & $70: 30$ & 1 & 20 \\
F6 & 0.05 & 2 & $70: 30$ & 1.5 & 20 \\
F7 & 0.05 & 2 & $55: 45$ & 1 & 20 \\
F8 & 0.05 & 2 & $55: 45$ & 1.5 & 20 \\
F9 & 0.05 & 2 & $55: 45$ & 2 & 20 \\
\hline
\end{tabular}

*S:L solid lipid to liquid lipid ratio. 


\section{Characterization of $R P G-N L C$}

\section{Particle size analysis}

The mean PS of the RPG-NLC and the particle size distribution expressed by the PDI of the prepared NLCs were determined using the dynamic light scattering (DLS) technique with Nanotrac particle size analyzer (Microtrac, USA). RPG-NLC samples were diluted by distilled water and the concentration of samples was adjusted for the optimal measurement condition. Three measurements were averaged for the mean PS and PDI and the standard deviation (SD) was calculated.

\section{Zeta potential analysis}

The measurement of the electric charge on the surface of the nanoparticles is a good indication of the physical stability of the most colloidal system (Dubey et al., 2012). The surface charge of the prepared RPG-NLC was measured by determining ZP on the nanoparticles surface. ZP was measured using Zetatrac W3547 (Microtrac, USA). Conductivity was in the range of 24-42 $\mu \mathrm{s} / \mathrm{cm}$ and the applied field strength was $10 \mathrm{kV} / \mathrm{m}$ with zeta run time of 30 seconds.

\section{Determination of entrapment efficiency and drug loading of RPG-loaded NLCs}

Both $\mathrm{EE} \%$ and DL\% were calculated by measuring the amount of free non-encapsulated RPG in the prepared RPG-NLC. Samples were centrifuged using cooling centrifuge (Centurion Scientific Ltd.,UK) as mentioned by Chalikwar et al. (2012) with small modifications. Briefly, $1.5 \mathrm{ml}$ of the NLCs dispersion is centrifuged while the speed of centrifuge was kept at 15,000 rpm for $60 \mathrm{~min}$ at temperature of $4^{\circ} \mathrm{C}$. After suitable dilution, the concentration of RPG in the supernatant was measured using spectrophotometer (Jenway 6305 spectrophotometer, China) at $\lambda_{\max } 237 \mathrm{~nm}$. EE\% and DL\% were calculated using the Equations 1 and 2 . The experiment was done in triplicates and the average and SD were calculated.

$$
\begin{aligned}
& \mathrm{EE} \%=\frac{\begin{array}{c}
\text { total weight of } \mathrm{RPG}-\text { weight of } \\
\text { RPG in supernatant }
\end{array}}{\text { total weight of } \mathrm{RPG}} \times 100 \\
& \mathrm{DL} \%=\frac{\mathrm{RPG} \text { in supernatant }}{\text { total weight of lipid }} \times 100
\end{aligned}
$$

\section{In vitro release study}

The in vitro RPG release from the RPG-NLC test was done using dialysis bag diffusion technique (Ebrahimi et al., 2015). Dialysis bags used was with a molecular weight cut off 12,000-14,000 were soaked in the release medium for 24 hours before use. Volume equivalent to $1 \mathrm{mg}$ of RPG of RPG suspension and RPG-NLC was added into a thoroughly sealed by double-folding on both sides dialysis bag, which was immersed in $50 \mathrm{ml}$ of simulated intestinal fluid (phosphate buffered saline, PBS, PH 6.8) at $37^{\circ} \mathrm{C} \pm 0.5^{\circ} \mathrm{C}$. Then, it was placed on shaking water bath at $50 \mathrm{rpm}$ (Wise ${ }^{\circledR}$ bathwater bath, Wised B, Germany) for 24 hours. At predefined time intervals, $1 \mathrm{ml}$ sample was withdrawn from dissolution medium and the amount of RPG released was analyzed spectrophotometrically at $\lambda_{\max } 237 \mathrm{~nm}$. The volume of dissolution medium was kept constant by the addition of replacement volumes of PBS after each sampling.

\section{Optimization and preparation of the optimized formula}

The relationship between responses and formulation variables of all prepared RPG-NLC were treated using a three-level factorial design model, which is a response surface technique done by the use of Statgraphics ${ }^{\circledR}$ Centurion software. These high, medium and low levels of variables $X_{1}$ and $X_{2}$ were selected from different preliminary experimentation. The optimization of variables was performed using the desirability function to obtain the levels of $X_{1}$ and $X_{2}$, which minimize $Y_{1}$ while maximizing $Y_{2}$ and $Y_{3}$.

\section{Characterization of the optimized formula}

The optimized prepared formula was characterized by determining PS, PDI, ZP and its morphology and size were studied using TEM technique. Then, the EE\% and DL\% were also calculated and the in vitro release behavior was studied and the best fitted kinetic model for the in vitro release was investigated. The crystallinity and polymorphism of the optimized formula were studied using the DSC technique.

\section{TEM}

The TEM measurement was done using the negative staining method as reported by Yu and Huang (2013), using electron microscope JTEM-1010, (JEOL ${ }^{\circledR}$, Tokyo, Japan). One drop of the dispersion of the RPG-NLC was put on a copper grid coating and the excess droplets were wiped using filter paper. After 5 minutes, one drop of uranyl acetate solution ( $2 \% \mathrm{w} / \mathrm{v})$ was then dropped onto the grids. After the samples were negatively stained and air-dried at room temperature, they were ready for the TEM investigation done at $74 \mathrm{kV}$.

\section{Kinetic study of the in vitro drug release of the optimized RPG- NLC formula}

The in vitro release of the RPG from the optimized formula was evaluated in comparison with the pure RPG. The dissolution medium and the conditions of the experiment were not changed from the conditions mentioned previously for the in vitro release of the nine prepared formulae.

RPG release data of the optimized formula were fitted to several mathematical models to know the RPG release mechanism from RPG-NLC in the simulated intestinal fluid. The models studied are zero-order model (percentage cumulative drug released vs. time), first-order model (log percentage cumulative drug remained vs. time), Higuchi diffusion model (percentage cumulative drug released vs. square root of time), and HixonCrowell model (cube root of percentage cumulative drug remaining $v s$. time) and the best-fitted model is the model with the highest correlation coefficient $(r)$.

\section{Differential scanning calorimetry analysis}

DSC analysis was done on the optimized formula, which was lyophilized to study the physical state and polymorphism of the RPG-NLC. The DSC measurements were performed using differential scanning calorimeter, Shimadzu DSC-50 (Japan). The four studied powders were the pure GMS, pure RPG, physical mixture in the ratio (1:1), and the lyophilized optimized RPGNLC formulae. In brief, samples of weight $2-5 \mathrm{mg}$ were heated, then scanned between $20^{\circ} \mathrm{C}$ and $250^{\circ} \mathrm{C}$. The heating rate was of 
$10^{\circ} \mathrm{C}$ minute $^{-1}$ and the measurements were done under nitrogen gas flow (30 ml minute ${ }^{-1}$ ) (Mazumder et al., 2017).

\section{RESULTS AND DISCUSSION}

\section{Particle size analysis}

Size of the nanoparticles plays a key role in the evaluation of the efficiency of nanoparticles for RPG delivery. The mean PS and PDI of RPG-NLC is listed in Table 3. The mean PS ranged from $182.7 \pm 07.9$ in formula F9 to $452.0 \pm 66.1$ in formula F2, while the narrowest PS distribution was in F7 with PDI of $0.09 \pm 0.06$, while the widest distribution with PDI of $0.54 \pm 0.01$ was in F5. The polynomial equation obtained by the linear regression of the data of particle size is shown in Equation 3

$$
\begin{aligned}
Y_{1}= & 365.55+6.65611 * X_{1}-282.233 * \\
& X_{2}+0.165111 * X_{1}^{2}-12.21 * \\
& X_{1} * X_{2}+216.0 * X_{2}^{2}
\end{aligned}
$$

Where $Y_{1}, X_{1}$, and $X_{2}$ are the particle size, liquid lipid concentration, and surfactant concentration, respectively.

As seen from the data illustrated in Figure $2 \mathrm{~A}$, the smallest PS obtained when the liquid lipid ratio was $45 \%$ with the higher concentration of tween 80 . This was in agreement with what stated in Kumar et al. (2013), who found that the highest percentage of tween 80 surfactant gave the lowest PS. Concerning the percentage of liquid lipid, Teeranachaideekul et al. (2008) found that the increase in the amount of liquid lipid should result in an increase in the mean PS of NLCs. Different findings were mentioned by Abdelbary and Haider (2011) who stated that there is no significant effect of liquid lipid to solid lipid ratio on the size of NLC formulae.

\section{Zeta potential}

RPG-NLC with high ZP is likely of better stability upon storage than the formulae of $\mathrm{ZP}$ value close to $0 \mathrm{mV}$. It is known that $\mathrm{ZP}$ values of more than \pm 30 are considered a good indication for the stability of the nanoparticles (Honary and Zahir, 2013). As shown in Table 3, Formula F7 showed the highest negative $\mathrm{ZP}$ value of $-44.4 \pm 6.25$, while $\mathrm{F} 2$ showed the smallest value of $-07.9 \pm 0.93$. The linear regression of the zeta potential data of the prepared formulae resulted in Equation 4

$$
\begin{aligned}
Y_{2}= & -15.9333+0.65 * X_{1}+33.4 * X_{2}+ \\
& 0.011037 * X_{1}^{2}-0.311111 * X_{1} * \\
& X_{2}-10.6667 * X_{2}^{2}
\end{aligned}
$$

Table 3. Mean PS, PDI, ZP, EE, and DL of RPG-NLC prepared formulae.

\begin{tabular}{cccccc}
\hline Form. & PS $(\mathbf{n m})$ & PDI & ZP $(\mathbf{m V})$ & EE $(\%)$ & DL $(\%)$ \\
\hline F1 & $214.4 \pm 33.6$ & $0.25 \pm 0.02$ & $-15.4 \pm 2.16$ & $81.9 \pm 0.95$ & $2.05 \pm 0.06$ \\
F2 & $452.0 \pm 66.1$ & $0.11 \pm 0.03$ & $-07.9 \pm 0.93$ & $78.7 \pm 1.23$ & $1.97 \pm 0.08$ \\
F3 & $281.9 \pm 12.4$ & $0.17 \pm 0.03$ & $-15.9 \pm 1.80$ & $74.7 \pm 0.82$ & $1.87 \pm 0.06$ \\
F4 & $313.0 \pm 09.8$ & $0.39 \pm 0.03$ & $-40.2 \pm 3.22$ & $85.5 \pm 0.95$ & $2.14 \pm 0.13$ \\
F5 & $195.2 \pm 20.8$ & $0.54 \pm 0.01$ & $-26.9 \pm 3.04$ & $84.2 \pm 2.14$ & $2.10 \pm 0.08$ \\
F6 & $312.0 \pm 37.6$ & $0.12 \pm 0.02$ & $-25.6 \pm 5.55$ & $80.2 \pm 1.66$ & $2.00 \pm 0.12$ \\
F7 & $410.0 \pm 42.2$ & $0.09 \pm 0.06$ & $-44.4 \pm 6.25$ & $82.6 \pm 2.06$ & $2.06 \pm 0.15$ \\
F8 & $229.0 \pm 48.2$ & $0.50 \pm 0.03$ & $-21.2 \pm 5.35$ & $81.0 \pm 1.18$ & $2.02 \pm 0.12$ \\
F9 & $182.7 \pm 07.9$ & $0.35 \pm 0.03$ & $-31.8 \pm 2.80$ & $78.9 \pm 1.98$ & $1.96 \pm 0.07$ \\
\hline
\end{tabular}

Where $Y_{2}, X_{1}$, and $X_{2}$ are the zeta potential, liquid lipid concentration, and surfactant concentration, respectively.

As seen from Figure 2B, no significant effect of Tween 80 concentration on the $\mathrm{ZP}$ value was observed. This might be due to the nature of Tween 80 as a non-ionic surfactant. It was noticed that formulae with higher content of OA in the RPG-NLC resulted in an increase in the $\mathrm{ZP}$ values and vice versa. This was suggested to be due to the presence of the carboxylic $\left(\mathrm{COO}^{-}\right)$ group in the OA. This was in complete agreement with the finding Teeranachaideekul et al. (2007) who stated that because of the negative charged medium chain triglycerides at their $\left(\mathrm{COO}^{-}\right)$ group, increasing the $\%$ of medium chain triglycerides increases the ZP of NLC, and in the absence of the solid lipid, the nanoemulsion showed the highest $\mathrm{ZP}$ values.

\section{Entrapment efficiency and drug loading of RPG-loaded NLCs}

The percentages of RPG entrapped in all RPG-NLC are listed in Table 3. From table 3 and figure $2 \mathrm{C}$, it is clear that high values of EE\% achieved and most of the RPG was entrapped in the nanoparticles. The lowest EE value was $74.7 \% \pm 0.82 \%$ in formula 3 with $30 \%$ OA and $2 \%$ Tween 80 , while the highest $\mathrm{EE} \%$ value was $85.5 \% \pm 0.95 \%$ in $\mathrm{F} 4$ which contains the lowest concentration of both liquid lipid and surfactant. As there is no change in the total amount of the lipids or the RPG, the ranking of the $\mathrm{DL} \%$ of the RPG-NLC formulae was the same as in EE\%. F4 showed the highest DL $\%$ with $2.14 \% \pm 0.13 \%$, while F3 had DL $\%$ of $1.87 \% \pm 0.06 \%$. It was found that high $\mathrm{EE} \%$ and $\mathrm{DL} \%$ values were obtained with low surfactant concentration. The polynomial equation of the entrapment efficiency is shown in Equation 5

$$
\begin{aligned}
Y_{3}= & 110.927-0.850667 * X_{1}-14.82 * X_{2}+ \\
& 0.00557037 * X_{1}^{2}+0.326222 * X_{1} * \\
& X_{2}-1.18667 * X_{2}^{2}
\end{aligned}
$$

Where $Y_{3}, X_{1}$, and $X_{2}$ are the entrapment efficiency, liquid lipid concentration, and surfactant concentration, respectively.

The highest three formulae in both $\mathrm{EE} \%$ and DL\% were with $1 \%$ Tween 80 . This may be due to the enhanced solubilization caused by Tween 80 in the aqueous phase. Luo et al. (2006) found the same findings and stated that when the amounts of the emulsifiers increased, the EE\% decreased due to the solubilization effect of the emulsifiers which enhance the solubility of the drug in the aqueous phase, not in the lipid nanoparticles. Same findings were reported by Venkateswarlu and Manjunath when they used poloxamer 188 as surfactant; as the percentage of poloxamer 188 increased, the $\mathrm{EE} \%$ was decreased marginally. This thought to be due to increased solubility of the drug used in the aqueous phase as the percentage of poloxamer 188 increased (Venkateswarlu and Manjunath, 2004).

\section{In vitro $R P G$ release from $R P G-N L C$ nanoparticles}

The in vitro release of RPG from the NLC compared to pure RPG powder was evaluated for 24 hours in simulated intestinal fluid ( $\mathrm{pH}$ 6.8). The concentrations of RPG were calculated using constructed calibration curve on RPG concentration range 5-50 $\mu \mathrm{g} / \mathrm{ml}$. The curve was linear and obeys the Beer's Lambert law over the whole concentration range, the equation describes the curve is $(y=0.02 x-0.0125)$ and the $r=0.9996$. The cumulative percentage released of RPG was illustrated in Figure 3. As seen from the graph, the release of RPG from all RPG-NLC formulae 

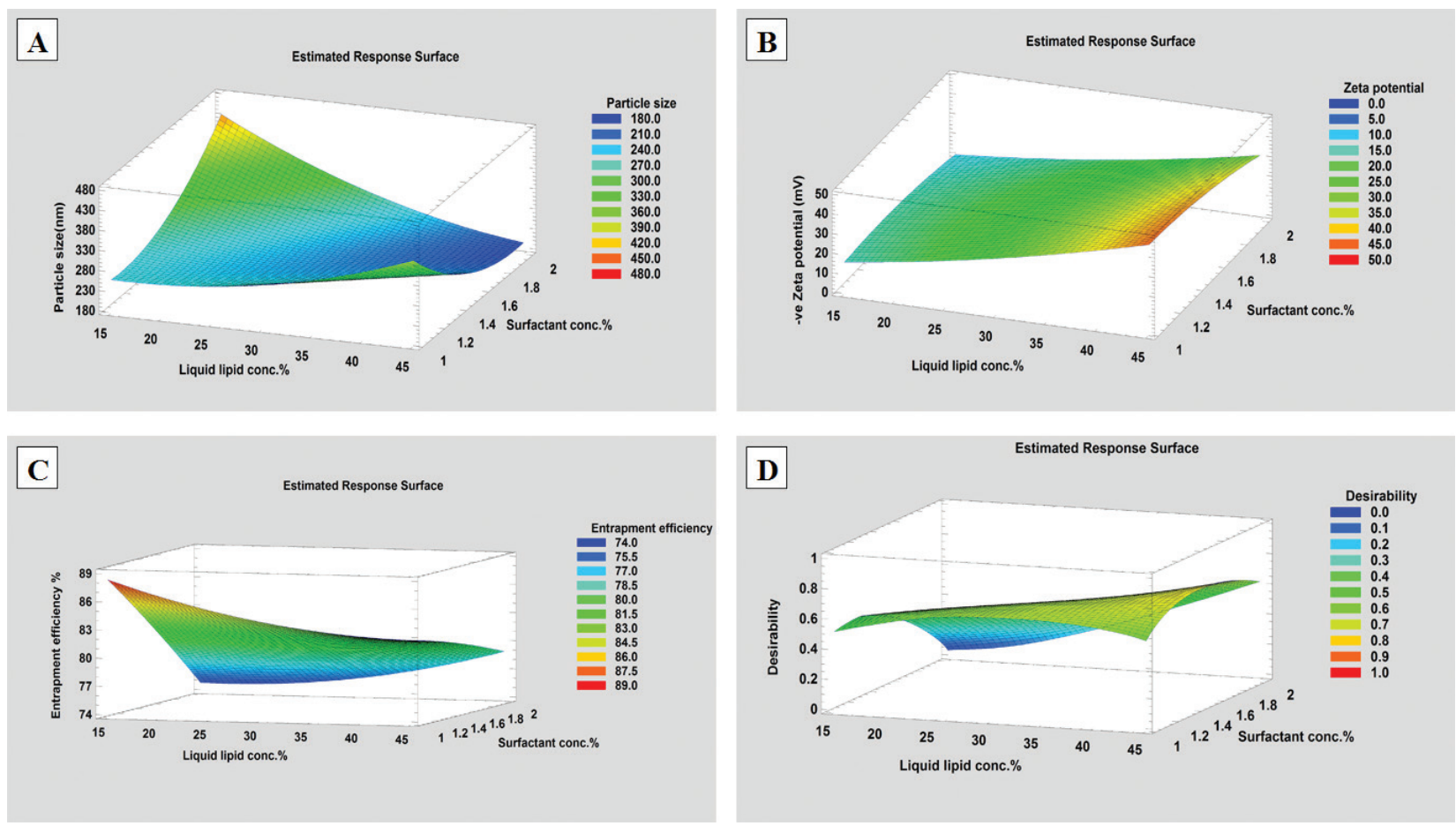

Figure 2. Response surface plots showing the effect of the liquid lipid concentration $[X 1]$ and surfactant concentration $[X 2]$ on $(\mathrm{A})$ particle size [Y1], (B) zeta potential [Y2], (C) entrapment efficiency [Y3], and (D) desirability function of the three responses [Y1, Y2 and Y3].

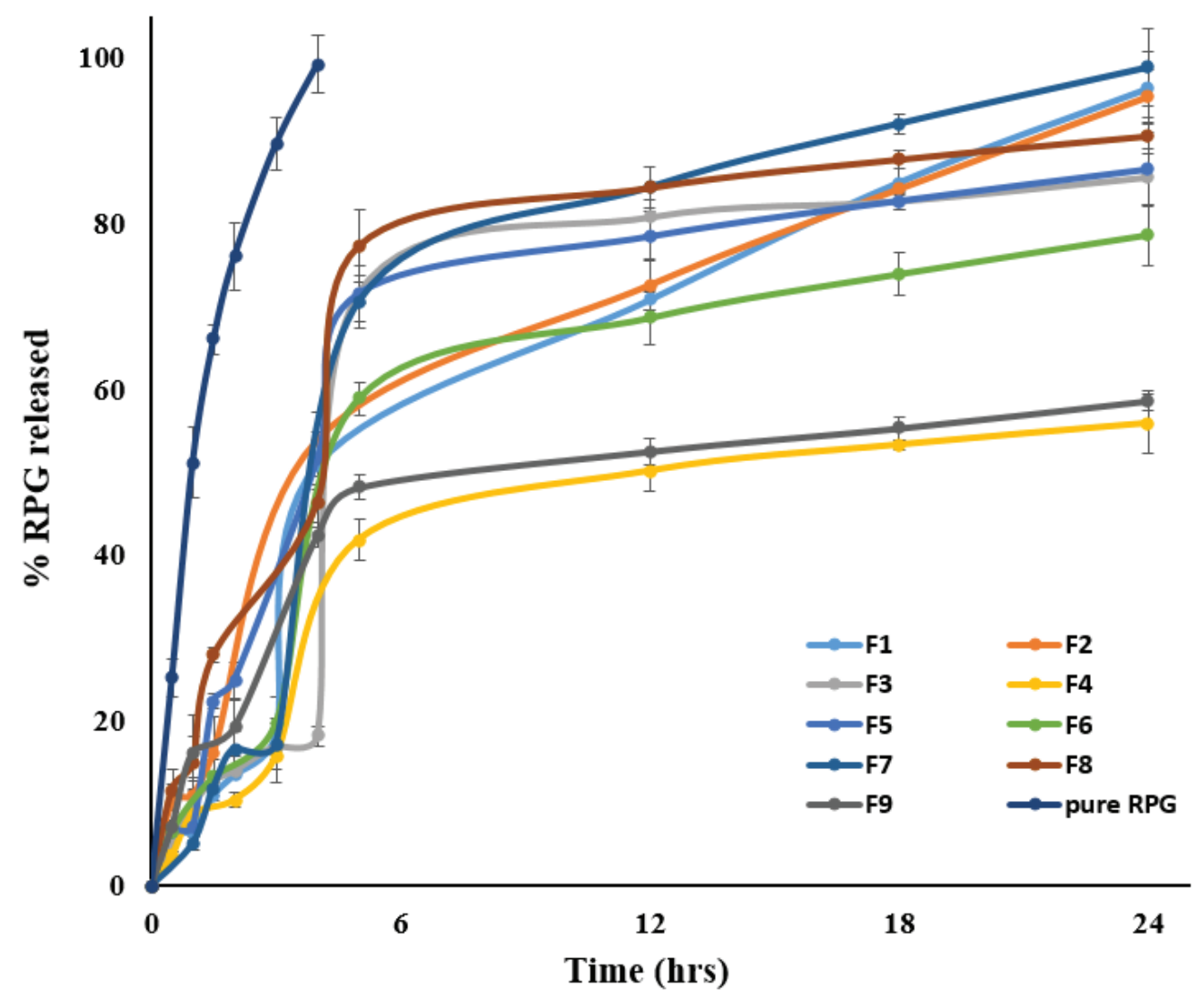

Figure 3. The in vitro release of RPG from the prepared RPG-NLC formulae compared to the pure RPG powder in the simulated intestinal fluid dissolution medium. 
was biphasic with the initial rapid release and sustained release in the second phase. The faster initial release was derived from the diffusion of RPG located at the surface of the nanoparticle, while the slower later one from that incorporated in the NLC core and released in a prolonged pattern via the erosion or degradation of the matrix (Zeng et al., 2012).

It is also clear that all formulae showed the slower release of the drug compared to the pure RPG. After 4 hours, the pure RPG dispersion released about $100 \%$, while all prepared formulae released much smaller amount of RPG within the same period. Formula F3 showed the lowest RPG release with only $18.125 \% \pm$ $1.15 \%$ of RPG, while F2 released the highest percentage of RPG $53.875 \% \pm 3.3 \%$. After 24 hours, the release of RPG from F4 was only $55.90 \% \pm 3.6 \%$, while the fastest RPG release from RPGNLC formulae was about $98.88 \% \pm 4.7 \%$ from formula F7. It is clear that the fastest release obtained in the formula with higher liquid lipid content, while the slowest release was when the OA is only $15 \%$. A possible explanation is that the presence of a high percentage of the lipid in the liquid state led to thermodynamic instability which enhances the release of the drug from the nanoparticles.

\section{Optimization of the independent variables}

The optimization of the concentration ratio of the liquid lipid to the solid lipid and the surfactant concentration was done using response surface methodology and through the determination of the desirability function.

This statistical function is considered one of the most important functions to optimize variables statistically. It is a function based on the concept that the quality of a formula that has many features is very unacceptable if one of them is outside of a desirable limit. It aims to adjust the formulation or process variable to values that ensure compliance with the criteria of all of the involved responses and so to provide the optimum value of compromise in the desirable joint response. This can be achieved by converting the multiple responses into a single response, then combining the individual responses into a composite function followed by its optimization (Vera Candioti et al., 2014).

All values of the optimized variables, predicted and observed responses are listed in Table 4. The optimum values of variables $X_{1}$ and $X_{2}$ were $45 \%$ and $1.476 \%$, respectively. The predicted responses were $242.5 \mathrm{~nm},-41.1 \mathrm{mV}$, and $81.1 \%$ for mean PS, ZP, and EE\%, respectively. The measurements done after the formulation of the optimized formula-as observed responses-were $228 \mathrm{~nm},-38.2 \mathrm{mV}$, and $80.2 \%$, respectively. Both ZP and EE\% showed a better observed response, while the predicted PS was smaller than that actually measured for the optimized formula, the mean PS and size distribution of the optimized formula is shown in Figure 4. All responses were close to the expected values and were within the statistically accepted deviation range. The desirability function for the optimization process equals 0.751189 (Figure 2D), this value is high enough to ensure acceptable values for the three studied responses.

\section{TEM}

The TEM photograph of the optimized RPG-NLC formula is shown in Figure 5 with magnification power
Table 4. The predicted and the observed response for the optimized RPG-NLC formula.

\begin{tabular}{lcc}
\hline Independent variables & \multicolumn{2}{c}{ Optimum value (\%) } \\
\hline$X_{1}$ Liquid lipid ratio & \multicolumn{2}{c}{45.0} \\
$X_{2}$ Surfactant concentration & \multicolumn{2}{c}{1.476} \\
Dependent variables & Predicted response & Observed response \\
$Y_{1}$ Particle size & $242.5 \mathrm{~nm}$ & $228 \mathrm{~nm}$ \\
$Y_{2}$ Zeta potential & $-41.1 \mathrm{mV}$ & $-38.2 \mathrm{mV}$ \\
$Y_{3}$ Entrapment efficiency & $81.1 \%$ & $80.2 \%$ \\
\hline
\end{tabular}

$80,000 \times$. From the photograph, it is clear that the NLC vesicles are separate entities. All particles appeared in the photograph were in the nanometer range. The TEM investigation also revealed that the nanoparticles of the RPG-NLC formula were homogeneous ellipsoidal or spherical in shape. This might indicate homogeneity and good uniformity of the release of the entrapped RPG. It was noticed that the vesicles look smaller in size when measured using TEM technique compared to that measured by DLS technique. As the preparation of the TEM sample involves drying, this leads to shrinkage of the nanoparticles so they appear smaller compared with the DLS measurement, in which the hydration of samples maintains the size of the nanoparticles (Sanad et al., 2010).

\section{Kinetic study of the in vitro drug release of the optimized RPG- NLC formula}

The in vitro release data of the optimized formula showed slower RPG release than all previously prepared NLC formulae. Optimized formula released only $17.13 \% \pm 0.53 \%$ of RPG after 4 hours, while after 24 hours, $44.38 \% \pm 2.1 \%$ of RPG released. This pattern of release proved that the optimized formulae achieved the controlled prolonged release pattern that allows sustained RPG release, hence better control of DM can be obtained.

Different kinetic models were applied to the RPG release data. RPG release from the optimized formula at $\mathrm{pH} 6.8$ did not show proper fitting to zero order, first order, and Hixon Crowell models and the $r$ values were $0.967,-0.979$, and 0.976 , respectively. The best-fitted model to describe the kinetics of the release and hence describe the release mechanism was Higuchi diffusion model with the highest $r$ value equals 0.992. Graphical representation of the application of Higuchi diffusion model to the release data is shown in Figure 6. The fitting of this model suggests that the major mechanism of the RPG release was by diffusion as this model best applied to systems shows release by diffusion through the lipid matrix (Siepmann and Peppas, 2011). This was in complete agreement with the findings of Sangsen et al. (2014), which prepared curcumin loaded NLC.

\section{Differential scanning calorimetry analysis}

DSC is an accurate, rapid, and reliable method for the characterization of crystallinity of the drug. It also gives a strong indication on the possible interactions that might occur between the drug and all additives, which is reflected in the thermogram by the appearance of new peaks, displacement of the endothermic peaks, or change in their shape or enthalpy (Maiti et al., 2007).

The thermograms of pure RPG, pure GMS, physical mixture of both, and the RPG-NLC optimized formula are shown in Figure 7. As seen from the figure, RPG showed a sharp 


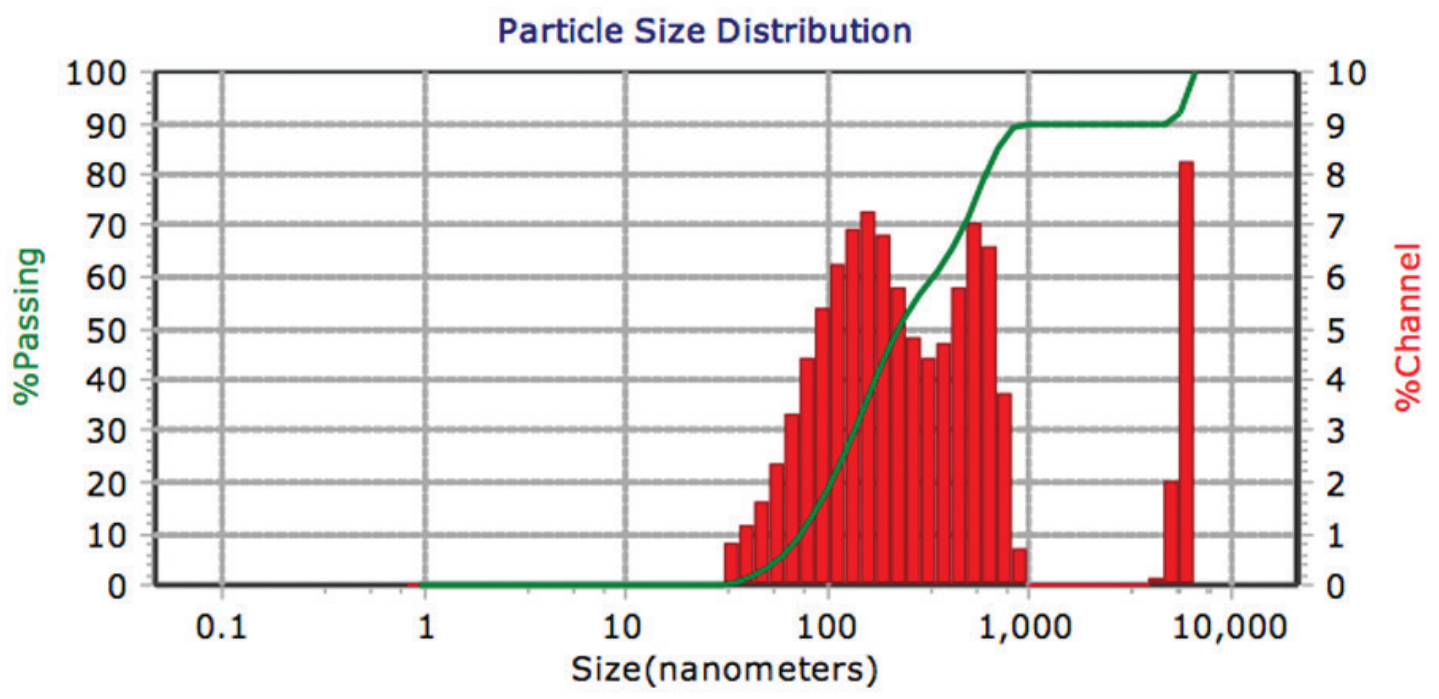

Figure 4. Particle size distribution of the optimized RPG-NLC formula.

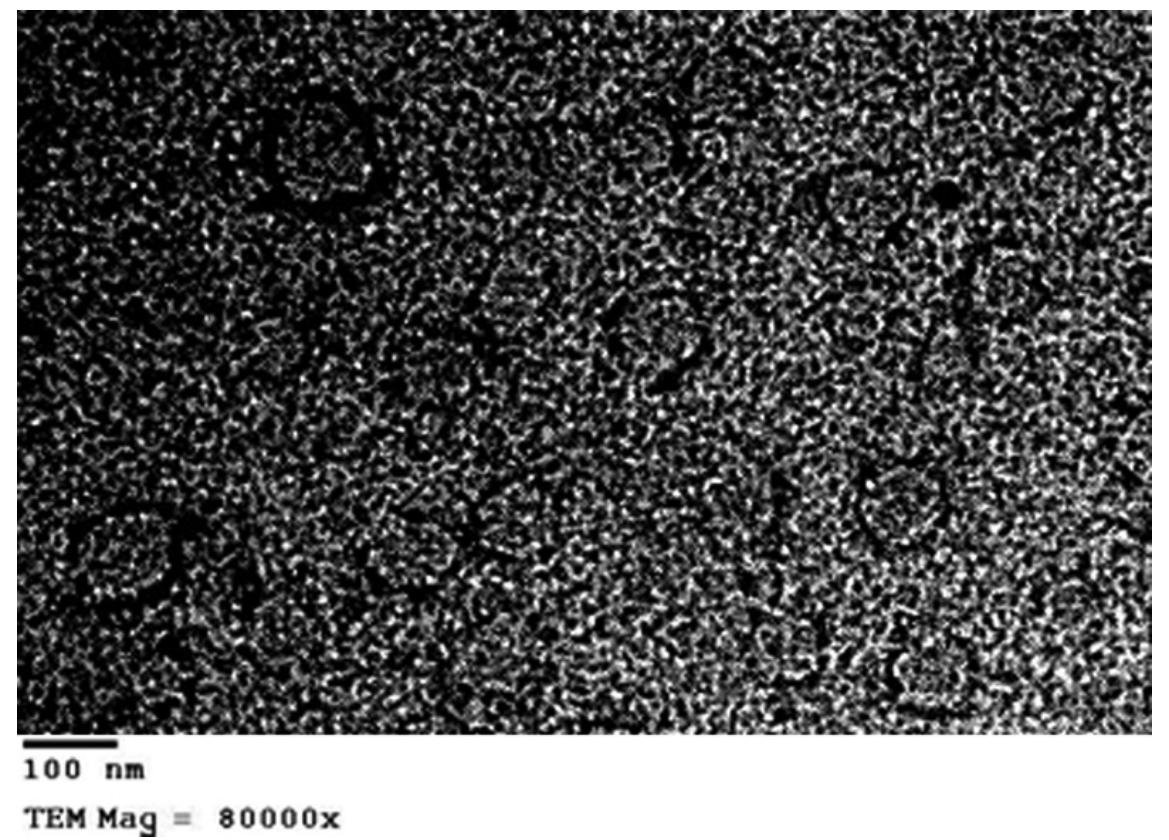

Figure 5. TEM photography of the lyophilized optimized RPG-NLC nanoparticles.

endothermic peak at $131.3^{\circ} \mathrm{C}$. This indicates that pure RPG is in the crystalline state and this value was in agreement with Purvis et al. (2007).

The GMS showed a sharp single endothermic peak at $69.1^{\circ} \mathrm{C}$. The physical mixture of the drug and the lipid showed two endothermic peaks at approximately the same temperatures for the pure powders. A slight widening in the peak of the RPG was noticed which might be an indication of the presence of slight affinity between RPG and GMS, but both are still in the crystalline state and no interaction was noticed. Complete disappearance of the melting peak of the RPG in its melting temperature range was noticed, while a slightly displaced peak for GMS appeared. This finding suggests the conversion of the crystalline RPG to its amorphous state. This signifies the possibility of interaction between the drug and the lipid and indicates a better solubility of the RPG which affects its release from the nanoparticles (Kassem et al., 2017).

\section{CONCLUSION}

In the current study, the RPG-NLC formulae were successfully prepared using emulsification-ultrasonification method. High RPG entrapment was achieved in the prepared nanosized vesicles. The liquid lipid and surfactant concentrations are crucial for optimizing size, charge, drug loading, drug entrapment, and RPG release from RPG-NLC. RPG-NLC achieved sustained controlled biphasic release in vitro, while the release kinetics of the optimized formula obeyed Higuchi diffusion kinetics model. RPG was in an amorphous state in the NLC, which allows better solubility and 


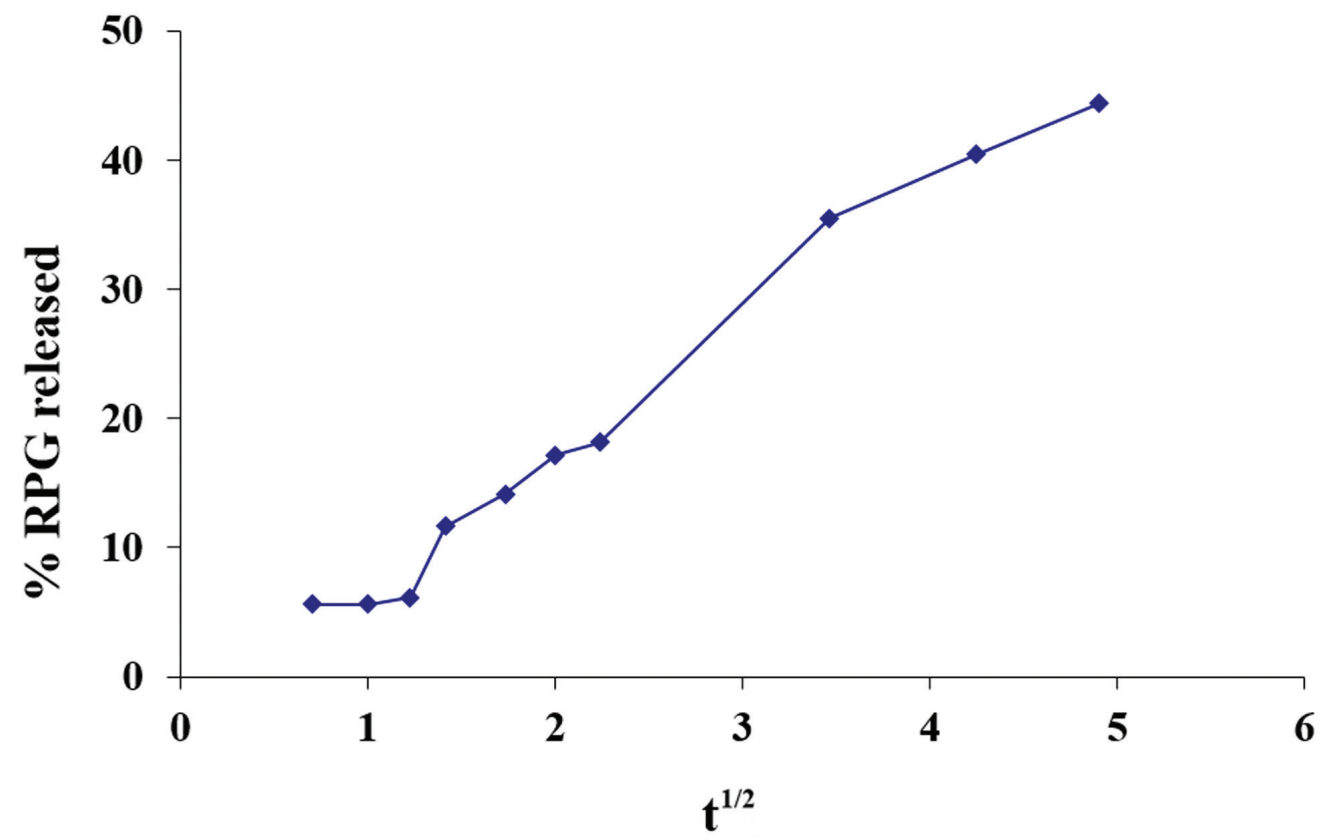

Figure 6. Higuchi diffusion model representation for the in vitro release of RPG from the optimized RPG-NLC formula.
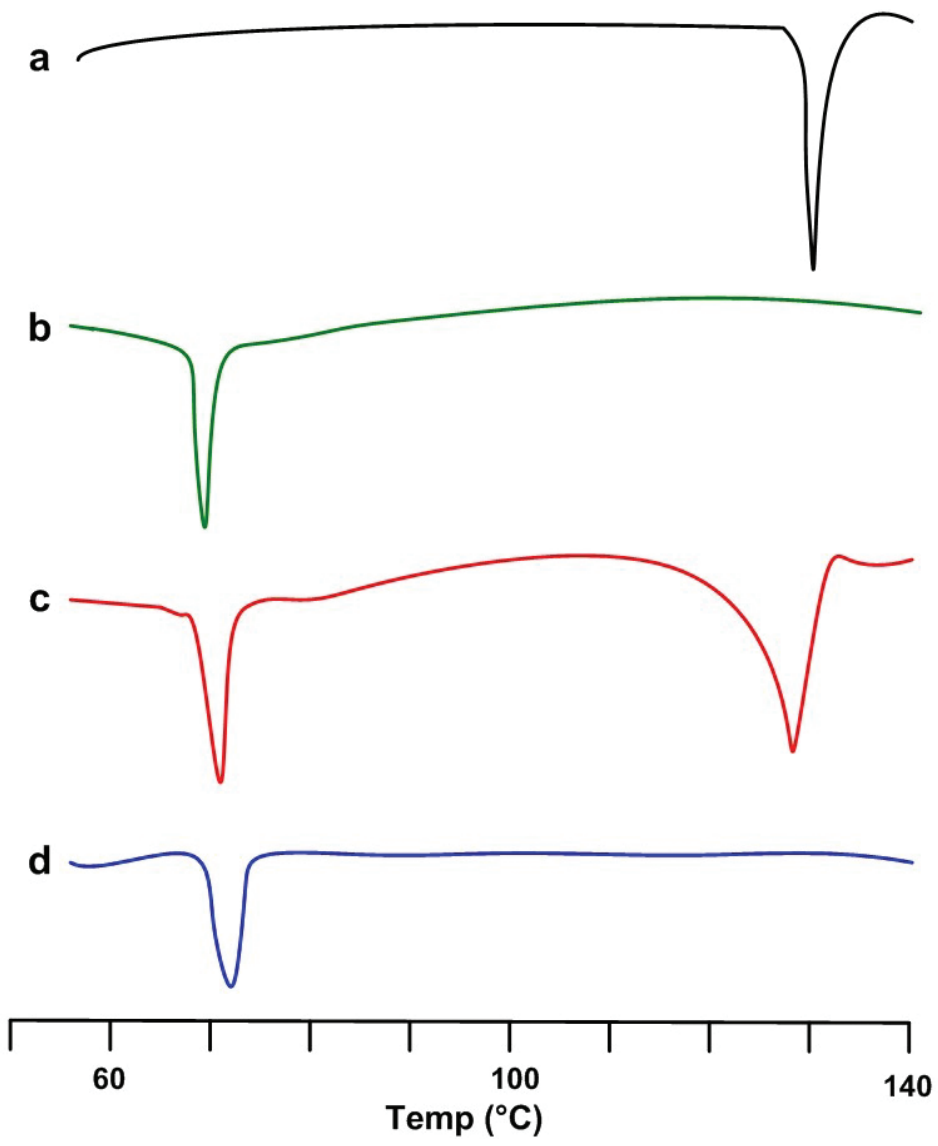

Figure 7. DSC thermograms of (A) pure RPG, (B) pure GMS, (C) physical mixture of RPG and GMS, and (D) optimized RPG-NLC formula. 
controlled release. Further studies on the in vivo pharmacodynamics, pharmacokinetic, and therapeutic effects must be done. Finally, NLC is a promising nanocarrier for the delivery of RPG orally.

\section{REFERENCES}

Abdelbary G, Haider G. In vitro characterization and growth inhibition effect of nanostructured lipid carriers for controlled delivery of methotrexate. Pharm Dev Technol, 2011; 18(5):1159-68.

Akhtar J, Fareed S, Aqil M. Stability-indicating assay of repaglinide in bulk and optimized nanoemulsion by validated high performance thin layer chromatography technique. J Pharm Bioallied Sci, $2013 ; 5(3): 184$

Akhtar J, Siddiqui HH, Fareed S, Badruddeen MK, Aqil M. Nanoemulsion: for improved oral delivery of repaglinide. Drug Deliv, 2016; 23(6):2026-34.

Chalikwar SS, Belgamwar VS, Talele VR, Surana SJ, Patil MU. Formulation and evaluation of nimodipine-loaded solid lipid nanoparticles delivered via lymphatic transport system. Colloids Surf B Biointerfaces, 2012; 97:109-16.

Das S, Chaudhury A. Recent advances in lipid nanoparticle formulations with solid matrix for oral drug delivery. AAPS PharmSciTech, 2011; 12(1):62-76

Das S, Ng WK, Kanaujia P, Kim S, Tan RBH. Formulation design, preparation and physicochemical characterizations of solid lipid nanoparticles containing a hydrophobic drug: effects of process variables. Colloids Surf B Biointerfaces, 2011; 88(1):483-89.

Dubey A, Prabhu P, Kamath JV. Nano structured lipid carriers: a novel topical drug delivery system. Int J PharmTech Res, 2011; 4(2):705-14.

Ebrahimi HA, Javadzadeh Y, Hamidi M, Jalali MB. Repaglinideloaded solid lipid nanoparticles: effect of using different surfactants/ stabilizers on physicochemical properties of nanoparticles. Daru, 2015; 23(1):46.

Gadadare R, Mandpe L, Pokharkar V. Ultra rapidly dissolving Repaglinide nanosized crystals prepared via bottom-up and top-down approach: influence of food on pharmacokinetics behavior. AAPS PharmSciTech, 2015; 16(4):787-99.

He W, Wu M, Huang S, Yin L. Matrix tablets for sustained release of Repaglinide: preparation, pharmacokinetics and hypoglycemic activity in Beagle dogs. Int J Pharm 2015; 478(1):297-307.

Honary S, Zahir F. Effect of zeta potential on the properties of nano-drug delivery systems - a review (Part 1). Trop J Pharm Res 2013; 12(2):255-64.

Hu S, Wang S, Fanelli B, Bell PA, Dunning BE, Geisse S, et al. Pancreatic beta-cell K (ATP) channel activity and membrane-binding studies with nateglinide: a comparison with sulfonylureas and Repaglinide. J Pharmacol Exp Ther, 2000; 293(2):444-52.

Kamble MS, Borwandkar VG, Bodade SS, Aute PP, Chaudhari PD, Bhosale AV. Optimization of self-nanoemulsifying drug delivery system (SNEDDS) of Repaglinide using D-optimal mixture experimental design. J Biomed Pharm Res, 2013; 2(3):100-8, 109.

Kassem, AA, Abd El-Alim SH, Basha M, Salama A. Phospholipid complex enriched micelles: a novel drug delivery approach for promoting the antidiabetic effect of Repaglinide. Eur J Pharm Sci, 2017; 99:75-84.

Kramer CK, Zinman B, Gross JL, Canani LH, Rodrigues TC, Azevedo MJ, et al. Coronary artery calcium score prediction of all cause mortality and cardiovascular events in people with type 2 diabetes: systematic review and meta-analysis. BMJ, 2013; 346(1):1654-4.

Kumar GSS, Talsania MP, Goli D, Karki R. Formulation and optimization of nanostructured lipid matrices of Repaglinide using factorial design. World J Pharm Pharm Sci, 2013; 2(6):5521-37.

Luo YF, Chen DW, Ren LX, Zhao XL, Qin J. Solid lipid nanoparticles for enhancing vinpocetine's oral bioavailability. J Control Release, 2006; 114(1):53-9.
Maiti K, Mukherjee K, Gantait A, Saha BP, Mukherjee PK Curcumin-phospholipid complex: preparation, therapeutic evaluation and pharmacokinetic study in rats. Int J Pharm, 2007; 330(1-2):155-63.

Mazumder S, Dewangan AK, Pavurala N. Enhanced dissolution of poorly soluble antiviral drugs from nanoparticles of cellulose acetate based solid dispersion matrices. Asian J Phar Sci, 2017; 12(6):532-41.

Purvis T, Mattucci ME, Crisp MT, Johnston KP, Williams RO. Rapidly dissolving Repaglinide powders produced by the ultra-rapid freezing process. AAPS PharmSciTech, 2017; 8(3):E58.

Sanad RA, Abdelmalak NS, Elbayoomy TS, Badawi AA Formulation of a novel oxybenzone-loaded nanostructured lipid carriers (NLCs). AAPS PharmSciTech, 2010; 11(4):1684-94.

Sangsen Y, Laochai P, Chotsathidchai P, Wiwattanapatapee R. Effect of solid lipid and liquid oil ratios on properties of nanostructured lipid carriers for oral curcumin delivery. Adv Mat Res, 2014; 1060:62-5.

Siepmann J, Peppas NA. Higuchi equation: derivation, applications, use and misuse. Int J Pharm, 2011; 418(1):6-12.

Swidan SA, Ghonaim HM, Samy AM, Ghorab MM. Efficacy and in vitro cytotoxicity of nanostructured lipid carriers for paclitaxel delivery. J Appl Pharm Sci, 2016; 6(9):018-26.

Tan SW, Billa N, Roberts CR, Burley JC. Surfactant effects on the physical characteristics of amphotericin B-containing nanostructured lipid carriers. Colloids Surf A Physicochem Eng Aspects, 2010; 372(1-3):73-9.

Teeranachaideekul V, Boonme $\mathrm{P}$, Souto EB, Müller RH, Junyaprasert VB. Influence of oil content on physicochemical properties and skin distribution of nile red-loaded NLC. J Control Release, 2008; 128(2):134-41.

Teeranachaideekul V, Souto EB, Junyaprasert VB, Müller RH. Cetyl palmitate-based NLC for topical delivery of coenzyme Q10 development, physicochemical characterization and in vitro release studies. Eur J Pharm Biopharm, 2007; 67(1):141-8.

Venkateswarlu V, Manjunath K. Preparation, characterization and in vitro release kinetics of clozapine solid lipid nanoparticles. J Control Release, 2004; 95(3):627-38.

Vera Candioti L, De Zan MM, Cámara MS, Goicoechea HC Experimental design and multiple response optimization. Using the desirability function in analytical methods development. Talanta, 2014; 124:123-38.

Weber S, Zimmer A, Pardeike J. Solid lipid nanoparticles (SLN) and nanostructured lipid carriers (NLC) for pulmonary application: a review of the state of the art. Eur J Pharm Biopharm, 2014; 86(1):7-22.

Yang Y, Corona A, Schubert B, Reeder R, Henson MA. The effect of oil type on the aggregation stability of nanostructured lipid carriers. J Colloid Interface Sci, 2014; 418:261-72.

Yu H, Huang Q. Bioavailability and delivery of nutraceuticals and functional foods using nanotechnology. Bio-Nanotechnol Revol Food Biomed Health Sci, 2013; 593-604.

Zeng N, Hu Q, Liu Z, Gao X, Hu R, Song Q, et al. Preparation and characterization of paclitaxel-loaded DSPE-PEG-liquid crystalline nanoparticles (LCNPs) for improved bioavailability. Int J Pharm, 2012; 424(1-2):58-66.

Zhu H, Zhang X, Li M-Z, Xie J, Yang X-L. Prevalence of type 2 diabetes and pre-diabetes among overweight or obese children in Tianjin, China. Diab Med J Br Diab Assoc, 2013; 30(12):1457-65.

\section{How to cite this article:}

Swidan SA, Mansour ZN, Mourad ZA, Elhesaisy NA, Mohamed NA, Bekheet MS, et al. DOE, formulation, and optimization of Repaglinide nanostructured lipid carriers. J App Pharm Sci, 2018; 8(10): 008-016. 